\title{
$\begin{array}{ll}\text { Research Square } & \text { Preprints are preliminary reports that have not undergone peer review. } \\ \text { They should not be considered conclusive, used to inform clinical practice, } & \text { or referenced by the media as validated information. }\end{array}$
}

\section{Ephrin-A2 promotes prostate cancer metastasis by enhancing angiogenesis and promoting EMT}

\section{Yao Zhao}

The second affiliated hospital of soochow university https://orcid.org/0000-0002-1831-5110

\section{Chenchen Cai}

medical technology school of xuzhou medical university

\section{Lubing Shi}

Xuzhou Medical University

Jiwei Wang

Xuzhou Medical University

\section{Miaomiao Zhang}

Xuzhou Medical University

Haoliang Zhang

Xuzhou Medical University

Ping Ma (D672443193@qq.com )

Affiliated Hospital of xuzhou medical University

\section{Shibao Li}

Affiliated Hospital of xuzhou Medical College

\section{Research}

Keywords: Prostate cancer, EphrinA2, Metastasis, Angiogenesis, EMT

Posted Date: September 24th, 2020

DOI: https://doi.org/10.21203/rs.3.rs-78710/v1

License: (c) (1) This work is licensed under a Creative Commons Attribution 4.0 International License.

Read Full License

Version of Record: A version of this preprint was published at Journal of Cancer Research and Clinical Oncology on March 27th, 2021. See the published version at https://doi.org/10.1007/s00432-021-036182. 


\section{Abstract \\ Background}

Ephrin-A2, a member of the Eph receptor subgroup, is used in diagnosing and determining the prognosis of prostate cancer. However, the role of ephrin-A2 in prostate cancer is still unclear.

\section{Methods}

We established stable clones overexpressing or silencing ephrin-A2 from prostate cancer cells. Then, CCK8 was used in analyzing the proliferation ability of cells. CD31 staining was used in evaluating angiogenesis. Migration and invasion assay were conducted in vivo and in vitro. The expression of EMTrelated markers was evaluated in prostate cancer cells through Western blotting.

\section{Results}

We revealed that the ectopic expression of ephrin-A2 in prostate cancer cells facilitated cell migration and invasion in vitro and promoted tumor metastasis and angiogenesis in vivo and that the silencing of ephrin-A2 completely reversed this effect. Although ephrin-A2 did not affect tumor cell proliferation in vitro, ephrin-A2 significantly promoted primary tumor growth in vivo. Furthermore, to determine the biological function of ephrin-A2, we assayed the expression of EMT-related markers in stable established cell lines. Results showed that the overexpression of ephrin-A2 in prostate cancer cells down-regulated the expression of epithelial markers (ZO-1, E-cadherin, and claudin-1) and up-regulated the expression of mesenchymal markers ( $\mathrm{N}$-cadherin, $\beta$-catenin, vimentin, Slug, and Snail), but the knocking out of ephrinA2 opposed the effects on the expression of EMT markers.

\section{Conclusions}

These findings indicate that ephrin-A2 promotes prostate cancer metastasis by enhancing angiogenesis and promoting EMT and may be a potentially therapeutic target in metastatic prostate cancer.

\section{Background}

Prostate cancer ( $\mathrm{PCa})$, one of the most common malignancies, poses threat to the health of men worldwide ${ }^{1}$. High metastasis and high recurrence rate after castration treatment are the dominant clinical features of PCa. Metastasis has become a constraint to the long-term survival of patients with PCa and is the key to conquering it. Therefore, a major clinical challenge is preventing PCa progression and metastasis. 
As the largest subgroup family of receptor tyrosine kinase, the Eph receptor family contains 14 members in mammals ${ }^{2}$. Ephrin-A2, belonging to Eph ligands, are widely expressed in normal tissues and has a vital function in tumors ${ }^{3-6}$ For example, ephrin-A2 is involved in breast carcinoma, liver cancer, and $\mathrm{PCa}^{4}, 5$. In addition, ephrin-A2 promotes the migration of MDA-MB-231 breast cancer cells and reduces E-cadherin expression ${ }^{6}$. We previously reported that ephrin-A2 expression is dramatically high in the tissues and exosomes derived from patients with $\mathrm{PCa}{ }^{7,8}$. Although ephrin-A2 has an ectopic expression in $\mathrm{PCa}$ patients, its function remains poorly investigated.

The abnormal activation of signaling pathways plays a significant role in tumor genesis, progression, and metastasis, and blocking key molecules is an approach for antitumor therapy. The Eph family can trigger EMT-related signaling pathways to regulate cancer ${ }^{9-11}$. EMT and its related markers, including $\mathrm{N}$ cadherin, vimentin, fibronectin, matrix metalloproteinases (MMPs), E-cadherin, $\beta$-catenin, Snail, and Slug, are associated with cancer development. When undergoing EMT, epithelial cells show polarity loss, changes in morphology and cytoskeletal organization, and dissolution of direct contacts. Hence, cell deterioration can be prevented by inhibiting the up-regulation of the expression of EMT-related markers, which might be novel anticancer therapeutic targets ${ }^{12}$.

In this study, we reported that ephrin-A2 stimulated tumor growth in vivo and promoted PCa angiogenesis and metastasis. We highlighted a novel method of using ephrin-A2 in altering the expression of EMTrelated markers to promote metastasis. Ephrin-A2 may be a potential target for cancer therapy because it regulates EMT-related markers. This study provides novel insights into the role ephrin-A2 in PCa tumorigenesis.

\section{Methods}

\section{Cell culture}

LNCaP, PC-3, and DU145 were purchased from American Type Culture Collection (ATCC). All the cell lines were cultured in DMEM/F-12 media (Gibco, USA), each containing 10\% fetal bovine serum (FBS, Gibco, USA), $100 \mathrm{U} / \mathrm{mL}$ penicillin, and $100 \mathrm{U} / \mathrm{mL}$ streptomycin, at $5 \% \mathrm{CO} 2,95 \%$ atmosphere, and $37^{\circ} \mathrm{C}$.

\section{Overexpression of ephrin-A2}

The full-length cDNA of human ephrin-A2 was cloned into lentiviruses vectors (pLent-U6-GFP-Puro) at certain site. Vectors within ephrin-A2 fragments or control were transfected into 293T cells at 70\% confluency for the generation of the lentiviruses. Then, the lentiviruses were transfected into PC-3 cells at appropriate confluency with polybrene for $24 \mathrm{~h}$. Subsequently, infected cells were selected with puromycin at $2 \mu \mathrm{g} / \mathrm{mL}$ for 1 week. Finally, the selected cells were harvested, and ephrin-A2 expression was confirmed through Western blotting.

\section{RNA interference of ephrin-A2}


Ephrin-A2 shRNA (shRNA-1, shRNA-2, and shRNA -3) or control shRNA were used for RNA interference. Briefly, lentivirus shRNA or control were transfected into the $293 \mathrm{~T}$ cells at $70 \%$ confluency to generate lentiviruses. Then, lentiviruses were transfected into LNCaP and DU145 cells at appropriate confluency with polybrene for $24 \mathrm{~h}$. Subsequently, the cells were selected with proper puromycin ( $\mathrm{LNCaP}$ for $1 \mu \mathrm{g} / \mathrm{mL}$, DU145 for $4 \mu \mathrm{g} / \mathrm{mL}$ ) for 1 week. Finally, the selected cells were harvested, and ephrin-A2 expression was confirmed through Western blotting.

\section{Cell proliferation}

A total of 3000 cells were plated and incubated for $24,48,72$, and 96 h. CCK-8 reagent (Keygen Biotech, Nanjing, China) was added to the media and cultured for $3 \mathrm{~h}$. The OD 450 value was measured with an automatic micro-plate reader every $24 \mathrm{~h}$ for 4 days for the generation of a growth curve.

\section{Evaluation of angiogenesis}

CD31 staining was used in evaluating microvascular density (MVD $)^{13}$. Briefly, the slides were immunohistochemical staining against CD31 (Servicebio Technology, Wuhan, China) were identified $(\times 100)$. Five areas of MVD were selected for counting manually $(\times 200)$. Brown-stained endothelial cells and their clusters that were clearly separated from adjacent microvessels, tumor cells, and other connective tissues were considered separate countable vessels. The mean score of the five hot spots was set as the level of MVD in each animal.

\section{Wound healing assay}

DU145, LNCaP and PC-3 cell lines were performed for wound healing assay. In brief, a scratch was made with a micropipette tip at $90 \%$ confluency. The wounded area was captured immediately after scratching and after $24 \mathrm{~h}$ with a microscope. Wound healing abilities were quantified by measuring the average gap area between 0 and $24 \mathrm{~h}$ with ImageJ.

\section{Migration assay}

Modified migration assays were performed as previously described ${ }^{14}$. Briefly, cells suspended in a $100 \mu \mathrm{L}$ serum-free medium were added to the up-wells, and the lower chambers contained $10 \%$ FBS as a chemoattractant. The plates were incubated for $24 \mathrm{~h}$. Then, the chamber membrane was fixed and stained with $0.1 \%$ crystal violet. Finally, the upper non-migrating cells were gently wiped off with cotton swabs. Six fields were counted randomly $(\times 200)$, and the average of three independent experiments was obtained.

\section{Invasion assay}

Modified invasion assays were performed as previously described ${ }^{14}$. Briefly, diluted Matrigel (Corning Costar, USA) with serum-free medium (1:5) was added to the upper chamber, and incubation was performed for $4 \mathrm{~h}$ at $37^{\circ} \mathrm{C}$. Cells suspended in a $100 \mu \mathrm{L}$ serum-free medium were added into the upper 
wells, and the lower chambers contained $10 \%$ FBS. The plates were incubated for $24 \mathrm{~h}$. Then, the well membrane was fixed and stained with $0.1 \%$ crystal violet. Finally, the upper non-migrating cells were gently wiped off with cotton swabs. Six fields were counted randomly $(\times 200)$, and the average of three independent experiments was used.

\section{Xenograft models}

Stable established cell lines were cultured in a DMEM/F-12 medium containing 10\% FBS, $100 \mathrm{U} / \mathrm{mL}$ penicillin, and $100 \mathrm{U} / \mathrm{mL}$ streptomycin at $5 \% \mathrm{CO} 2,95 \%$ atmosphere, and $37^{\circ} \mathrm{C}$. Single cell suspensions with a concentration of $5 \times 10^{7}$ cells were inoculated into nude mice at one side of the abdomen. Six $\mathrm{BALB} / \mathrm{C}$ (nu/nu) nude mice aged 4-6 weeks were randomly divided into two groups of three. The inoculation site was observed, and whether the site was infected or not and whether the tumor had subsided naturally were checked daily. The tumor long diameter (a) and short diameter (b) were measured every week, then the tumor volume was calculated according to the formula: volume $=$ length $\times$ width $^{2}$ / 2 for the drawing of a growth curve. After 7 weeks, nude mice were sacrificed, and tumor tissues, forelimb axillary lymph nodes, and lung tissues were obtained, and tumor masses were weighed. The tumor tissues, left axillary lymph nodes, and left lung tissue were subjected to immunohistochemistry and HE staining. Paraffin sections were subjected to staining, and blood vessel density was assayed with Image Plus.

\section{PCR-based detection of human Alu sequences}

Human disseminated tumor cells (DTCs) were detected in lymph nodes and lungs with Alu-qPCR as previously described ${ }^{15}$ under the following conditions: $95^{\circ} \mathrm{C}$ for $10 \mathrm{~min}, 40$ cycles at $95^{\circ} \mathrm{C}$ for $5 \mathrm{~s}, 65^{\circ} \mathrm{C}$ for $5 \mathrm{~s}$, and $72^{\circ} \mathrm{C}$ for $20 \mathrm{~s}$. Numerical data were determined against a standard curve established using murine blood DNA containing log-fold dilutions of DNA from $1 \times 10^{6}$ prostate carcinoma cells $\triangle P C-3$ or DU145囚grown in culture. Negative controls were established for each tissue by testing DNA from species with similar sex and age. The independent experiments were repeated at least three times.

\section{Immunohistochemistry}

Immunohistochemical staining was performed according to a standard method. Briefly, primary tumor tissues were fixed in a 10\% formalin buffer, embedded in paraffin, and sectioned at $4 \mu \mathrm{m}$. Then, the slides were deparaffinized, rehydrated, and microwaved for $30 \mathrm{~min}(\mathrm{pH} \mathrm{6.0)}$ for the retrieval of antigen epitopes in citrate buffer. The slides were subsequently treated with $3 \% \mathrm{H}_{2} \mathrm{O}_{2}$ for 10 min for the elimination of endogenous peroxidase. The slides were incubated overnight with a primary antibody (E-cadherin, $\mathrm{N}$ cadherin, CD31). The section were washed with PBS and then incubated with secondary antibody for 30 min at room temperature. 3,3'-Diaminobenzidine (DAB) was used for color detection, and nuclei were counterstained with hematoxylin.

\section{Western blotting assay}


Total protein was extracted from cells, and protein concentrations were determined using a BCA protein reagent kit (Beyotime, Nantong, China). Lysates were separated and transferred to a polyvinylidene fluoride membrane (Millipore, Bedford, USA) and incubated with ephrin-A2 (Affinity Biosciences, Cincinnati, USA) and EMT primary antibodies (Cell Signaling Technology, Danvers, USA) at $4{ }^{\circ} \mathrm{C}$ for overnight. The membranes were washed three times, incubated with secondary antibodies for $2 \mathrm{~h}$, and visualized using an enhanced chemiluminescent substrate (Millipore, Bedford, USA). Finally, the membranes were visualized again using another enhanced chemiluminescent substrate (Bio-Rad Laboratories Inc, California, USA).

\section{Statistical analysis}

SPSS16.0 (SPSS, Chicago, USA) was used in statistical analyses. Multiple groups were evaluated with one-way analysis of variance (ANOVA), Statistical between groups were determined through $t$-test. A $P$ value of less than 0.05 was considered statistically significant.

\section{Results}

\section{Ephrin-A2 expression in stable established PCa cell lines}

To explore ephrin-A2 exerted function in PCa, we first developed stable clones overexpressing or knocking down ephrin-A2 in PCa cell lines. The expression of ephrin-A2 in established cell lines was confirmed through Western blotting. Ephrin-A2 showed forced expression in overexpressed PC-3 cells. (Fig. 1A). For the purpose of the efficiency of inhibition, we selected three RNA interferences to block ephrin-A2 expression. Compared with shRNA-1 and shRNA-2 vectors, ephrin-A2 showed significantly inhibited expression in the DU145 and LNCaP cells infected with shRNA-3 lentiviral particles (Fig. 1B and C). Therefore, we used cells infected with shRNA-3 lentiviral particles for the next step.

\section{Ephrin-A2 contributes to tumor growth}

To investigate whether ephrin-A2 functions in tumor growth, we established stable overexpressing ephrinA2 in PC-3 cells and silencing ephrin-A2 in DU145 and LNCaP, respectively. No significant difference in proliferation between the control and experimental group was observed in vitro (Fig. 2A). To further verify the feature of this effect, a subcutaneous xenograft model in mice was established. Ephrin-A2 overexpression in the PC-3 cells generated xenografts that were larger than those in the control, consistent with the silencing of ephrin-A2 cells (Fig. 2B). This result indicates that ephrin-A2 contributes to tumor growth in vivo, rather than to proliferation in vitro.

\section{Ephrin-A2 promotes tumor angiogenesis}

Ephrin-A2 may affect tumor environments. By determining whether ephrin-A2 plays a role in tumor angiogenesis, we found that the level of CD31, an indicator of angiogenesis, showed a higher increase in the PC-3 group with overexpressed ephrin-A2 than that in the control (Fig. 3). The silencing of ephrin-A2 in 
the DU145 group decreased microvascular density. The data indicate that ephrin-A2 may promote tumor angiogenesis to increase tumor growth.

\section{Ephrin-A2 led to tumor metastasis}

To evaluate the metastasis potential of ephrin-A2, we examined the effect of ephrin-A2 on migration in PC-3 cells. The overexpression of ephrin-A2 in the PC-3 cells facilitated migration (Supporting Fig. 1 and Fig. 4A, $P<0.001)$. Invasion assay showed that the overexpression of ephrin-A2 in PC-3 cells promoted the migration of cells (Fig. 4B, $P<0.001$ ). These data show that ephrin-A2 can promote tumor invasion in $\mathrm{PC}-3$ cells. To further verify whether ephrin-A2 led to invasion in $\mathrm{PCa}$, we performed migration and invasion assays in depletion of ephrin-A2 in LNCaP and DU145 cells. We found that Ephrin-A2 enhanced the capability of migration and invasion in LNCaP cells (Fig. 4A and Fig. 4B, $P<0.001$, Supporting Fig.2) and that the loss of ephrin-A2 significantly inhibited DU145 cell invasion (Fig. 4B). Nevertheless, no significantly changes in cell migration ability was observed in the DU145 cells (Supporting Fig. 3 and Fig. $4 \mathrm{~A}, P>0.05)$. These data suggest that ephrin-A2 promotes PC-3, LNCaP, and DU145 migration and invasion but has no effect on the migration capabilities of DU145.

Furthermore, we assessed whether ephrin-A2 can promote metastasis in vivo in a subcutaneous xenograft model using PC-3 and DU145 cells in nude mice. Tissues harvested from overexpressing ephrin-A2 PC-3 groups and ephrin-A2 knock-down DU145 group. HE staining showed that ephrin-A2 significantly increased the incidence of metastases to local draining lymph nodes (LN) and lungs compared with that in the control group (Fig. 4C and Fig. 4D, $P<0.01$ ). Collectively, these observations indicate that ephrin-A2 contributes to tumor metastasis.

\section{Ephrin-A2 regulates EMT related markers}

Ephrin-A2, as a member of the Eph receptor tyrosine kinase ligand family, regulates a series of downstream molecules. To determine whether the promotion of metastasis by ephrin-A2 is associated with the EMT family, we tested the expression of EMT-related markers (ZO-1, N-cadherin, E-cadherin, $\beta$ catenin, vimentin, Slug, Snail, and claudin-1) in stable PCa cells through Western blotting. The results show the down-regulated expression of epithelial markers (ZO-1, E-cadherin, and claudin-1) and upregulated expression of mesenchymal markers ( $\mathrm{N}$-cadherin, $\beta$-catenin, vimentin, Slug, and Snail), but the knocking out of ephrin-A2 opposed the effect on the expression of EMT markers (Fig. 5). These data indicate that ephrin-A2 medicated tumor cell metastasis may be associated with the activation of EMT progress.

To confirm the hypothesis, subcutaneous xenograft mouse model was used. The mice were sacrificed, and related tissues were harvested for E-cadherin and N-cadherin expression analyses. In the overexpressing ephrin-A2 PC-3 groups, E-cadherin was down-regulated compared with that in the control group, whereas N-cadherin was up-regulated (Fig. 6A and 6B). Similarly, the depletion of ephrin-A2 expression was consistent with a previous result (Fig. 6A and 6B). The results further show that the activation of EMT signaling pathway play a vital role in ephrin-A2 medicated PCa metastasis. 


\section{Discussion}

In this study, we first developed stable established cell lines with overexpressing and knock-down, and our results demonstrated that ephrin-A2 promotes prostate cancer metastasis and angiogenesis. Furthermore, we provided novel insights into this mechanism, by which ephrin-A2 alters EMT-related markers to promote cancer metastasis. Moreover, ephrin-A2 may be a potential target for cancer metastasis therapy because it regulates EMT-related markers.

In our previous reports, we found that ephrin-A2 expression is significantly higher in the tissues and exosomes derived from patients with $\mathrm{PCa}^{7,8}$. However, in the present study, ephrin-A2 overexpression did not affect cell proliferation in PC-3 cell lines in vitro, and the depletion of ephrin-A2 in DU145 and LNCaP confirmed this finding. Ephrin-A2 contributed to tumor growth in vivo, and this result is consistent with the proliferation of liver cancer cells ${ }^{5}$. These results show that ephrin-A2 may affect cells in tumor environments and promotes tumor growth, rather than in the tumors. Traditionally, tumor growth depends on multiple factors, such as blood vessel development, growth factors, and nutrition supply. Many studies reported that the Eph family is involved in tumor angiogenesis ${ }^{16-18}$. To further elucidate the biological functions of ephrin-A2, we detected CD31, an indicator of angiogenesis. Our results show that ephrin-A2 contribute to tumor growth in vivo, rather than in vitro, suggesting that tumor proliferation rate is not responsible for tumor growth. Simultaneously, the depletion of ephrin-A2 reduced angiogenesis. Hence, we speculated that ephrin-A2 can induce angiogenesis and promote tumor metastasis. Overall, these observations revealed that ephrin-A2 may promote PCa progression and metastasis by enhancing angiogenesis, at least in part.

Typically, an important feature of malignant tumor cells is invasion. In breast tumor cells, the ephrin-A2 gene promotes cell migration. Feng et $a t^{5}$ confirmed that ephrin-A2 can promote liver cancer cell invasion in vivo. Consistent with previous studies, our study also found that ephrin-A2 has a positive action on cell invasion in $\mathrm{PCa}$ in vitro and in vivo. Our results show that the activity of ephrin-A2 can enhance cell motility and invasion. Further, ephrin-A2 increased the incidence of metastases in lymph nodes (LN) and lungs in vivo, indicating that ephrin-A2 leads to tumor metastasis.

EMT, an important mechanism of invasion and metastasis, has been observed in many malignant tumors in humans, but the pathogenesis of this disease remains unclear ${ }^{19}$. Zheng et $a^{20}$ found that after knocking out the CCAT2 gene, cell proliferation, invasion, and migration were significantly enhanced, suggesting that EMT is closely related to PCa invasion and metastasis. In our study, EMT-related markers were evaluated in stable established cell lines through Western blotting. Our studies identified that ephrinA2 can inhibit E-cadherin, ZO-1, and claudin-1 expression and enhance $\beta$-catenin, vimentin, Snail, Slug, and $\mathrm{N}$-cadherin. E-cadherin is involved in the progression of human malignancies progression, and the low expression of E-cadherin is associated with lymphatic vessel invasion ${ }^{10,21}$. When EMT occurs, epithelial cells lose polarity and show enhanced ability to migrate and promote cytoskeletal rearrangement. The overexpression of Eph receptor promotes migration and invasion and inhibits Ecadherin-independent ligand-dependent mechanisms through the downstream singling pathways 22,23 . 
This study reveals that ephrin-A2 down-regulates E-cadherin and loss cell adhesion to promote invasion and metastasis, suggesting that E-cadherin has potential as a tumor suppressor.

In many tumors, $\mathrm{N}$-cadherin is overexpressed and is related to metastasis ${ }^{24,25}$. E-cadherin and $\mathrm{N}$-cadherin belong to the cadherin family. E-cadherin loss is always accompanied by the up-regulation of $\mathrm{N}$-cadherin, which indicates poor prognosis ${ }^{26}$. $\mathrm{N}$-cadherin promotes cancer metastasis to regulate adhesion and angiogenesis ${ }^{27,28}$. Owing to the low expression of $\mathrm{N}$-cadherin in DU145, we assayed $\mathrm{N}$-cadherin in the overexpression ephrin-A2 group only. In this study, ephrin-A2 can increase $\mathrm{N}$-cadherin expression but down-regulate E-cadherin. These data indicate that ephrin-A2 promotes PCa metastasis and angiogenesis by regulating EMT-related markers.

$\mathrm{N}$-cadherin and vimentin not only promote tumor invasion and are associated with poor prognosis but also are therapeutic targets in cancer ${ }^{11,29,30}$. Our study indicates that ephrin-A2 intensifies the expression of $\mathrm{N}$-cadherin and vimentin, thereby accelerating tumor invasion and resulting in poor prognosis. Thus, ephrin-A2 is a promising target for PCa cancer therapy.

Another key factors, Snail and Slug, are linked to the promoter of E-cadherin and directly involved in inhibiting the expression of E-cadherin ${ }^{31,32}$. Ephrin-A2 probably interferes with Snail and Slug to downregulate E-Cadherin.

In conclusion, we demonstrated that ephrin-A2 promotes $\mathrm{PCa}$ metastasis and angiogenesis by altering EMT-related markers. Furthermore, ephrin-A2 may be a potential target for cancer metastasis therapy because it regulates EMT-related markers.

\section{Conclusion}

In our study, we revealed that EphrinA2 facilitated cell migration and invasion in vitro and promoted tumor metastasis and angiogenesis in vivo. Furthermore, EphrinA2 could down-regulated the expression of epithelial markers and up-regulated the expression of mesenchymal markers. These above findings indicate that EphrinA2 promotes prostate cancer metastasis by enhancing angiogenesis and promoting EMT and may be a potentially therapeutic target in metastatic prostate cancer.

\section{Abbreviations}

PCa: prostate cancer; EMT:epithelial-mesenchymal transition; MMPs:matrix metalloproteinases; FBS:fetal bovine serum; MVD:microvascular density; LN:lymph nodes.

\section{Declarations}

\section{Availability of data and materials}


The raw data used and analyzed in the current study are available from the corresponding author upon a reasonable request.

\section{Ethics approval and consent to participate}

The animals in this study were obtained in accordance with the ethical standards of the ethics committee of the affiliated Hospital of Xuzhou Medical University.

\section{Consent for publication}

Not applicable

\section{Competing interests}

The authors declare that they have no competing interests

\section{Funding}

This study was supported by the Jiangsu Provincial Medical Youth Talent (No. QNRC2016781) and the National Natural Science Foundation of China (No. 81802094) and Postdoctoral Science Foundation of China (No. 2020M670076ZX)

\section{Authors' contributions}

SBL, YZ and CCC contributed to the idea, drafting and editing of the manuscript. LUS and JWW completed statistical analysis. MMZ and HLZ contributed to literature search and feeding animals and dataanalysis. PM and SBL revised the manuscript with critical reviews and comments and all the authors approved the final version.

\section{Acknowledgements}

Not applicable

\section{References}

1. Smith RA, Manassaram-Baptiste D, Brooks D, et al. Cancer screening in the United States, 2014: a review of current American Cancer Society guidelines and current issues in cancer screening. CA Cancer J Clin. 2014;64(1):30-51.

2. Manning G, Whyte DB, Martinez R, Hunter T, Sudarsanam S. The protein kinase complement of the human genome. Science. 2002;298(5600):1912-34. 
3. Hafner C, Schmitz G, Meyer S, et al. Differential gene expression of Eph receptors and ephrins in benign human tissues and cancers. Clin Chem. 2004;50(3):490-9.

4. Fox BP, Kandpal RP. Invasiveness of breast carcinoma cells and transcript profile: Eph receptors and ephrin ligands as molecular markers of potential diagnostic and prognostic application. Biochem Biophys Res Commun. 2004;318(4):882-92.

5. Feng YX, Zhao JS, Li JJ, et al. Liver cancer: EphrinA2 promotes tumorigenicity through Rac1/Akt/NFkappaB signaling pathway. Hepatology. 2010;51(2):535-44.

6. Zhong Shi X, Wang YX, et al. A Primary Study on ephrin-A2 Promoted Migration of MDA-MB-231 Breast Cancer Cells. Journal of Medical Research, 2015,44(9).

7. Li S, Wu Z, Chen Y, et al. Diagnostic and prognostic value of tissue and circulating levels of Ephrin-A2 in prostate cancer. Tumour Biol. 2016;37(4):5365-74.

8. Li S, Zhao Y, Chen W, et al. Exosomal ephrinA2 derived from serum as a potential biomarker for prostate cancer. J Cancer. 2018;9(15):2659-65.

9. Hou F, Yuan W, Huang J, et al. Overexpression of EphA2 correlates with epithelial-mesenchymal transition-related proteins in gastric cancer and their prognostic importance for postoperative patients. Med Oncol. 2012;29(4):2691-700.

10. Yuan W, Chen Z, Wu S, et al. Expression of EphA2 and E-cadherin in gastric cancer: correlated with tumor progression and lymphogenous metastasis. Pathol Oncol Res. 2009;15(3):473-8.

11. Macrae M, Neve RM, Rodriguez-Viciana P, et al. A conditional feedback loop regulates Ras activity through EphA2. Cancer Cell. 2005;8(2):111-8.

12. Lahat G, Zhu QS, Huang KL, et al. Vimentin is a novel anti-cancer therapeutic target; insights from in vitro and in vivo mice xenograft studies. PLoS One. 2010;5(4):e10105.

13. Foote RL, Weidner N, Harris J, et al. Evaluation of tumor angiogenesis measured with microvessel density (MVD) as a prognostic indicator in nasopharyngeal carcinoma: results of RTOG 9505. Int J Radiat Oncol Biol Phys. 2005;61(3):745-53.

14. Su B, Bu Y, Engelberg D, Gelman IH. SSeCKS/Gravin/AKAP12 inhibits cancer cell invasiveness and chemotaxis by suppressing a protein kinase C- Raf/MEK/ERK pathway. J Biol Chem. 2010;285:4578-86.

15. Liu W, Guan M, Hu T, Gu X, Lu Y. Re-expression of AKAP12 inhibits progression and metastasis potential of colorectal carcinoma in vivo and in vitro. PLoS One. 2011;6:e24015.

16. Pasquale EB. Eph receptors and ephrins in cancer: bidirectional signalling and beyond. Nat Rev Cancer. 2010;10(3):165-80.

17. Nassar ZD, Moon H, Duong T, et al. PTRF/Cavin-1 decreases prostate cancer angiogenesis and lymphangiogenesis. Oncotarget. 2013;4(10):1844-55.

18. Kandouz M. The Eph/Ephrin family in cancer metastasis: communication at the service of invasion. Cancer Metastasis Rev. 2012. 31(1-2): 353 - 73. 
19. Colas E, Pedrola N, Devis L, et al. The EMT signaling pathways in endometrial carcinoma. Clin Transl Oncol. 2012;14(10):715-20.

20. Zheng J, Zhao S, He X, et al. The up-regulation of long non-coding RNA CCAT2 indicates a poor prognosis for prostate cancer and promotes metastasis by affecting epithelial-mesenchymal transition. Biochem Biophys Res Commun. 2016;480(4):508-14.

21. Saito T, Masuda N, Miyazaki T, et al. Expression of EphA2 and E-cadherin in colorectal cancer: correlation with cancer metastasis. Oncol Rep. 2004;11(3):605-11.

22. Fang WB, Ireton RC, Zhuang G, Takahashi T, Reynolds A, Chen J. Overexpression of EPHA2 receptor destabilizes adherens junctions via a RhoA-dependent mechanism. J Cell Sci. 2008;121(Pt 3):35868.

23. Miura K, Nam JM, Kojima C, Mochizuki N, Sabe H. EphA2 engages Git1 to suppress Arf6 activity modulating epithelial cell-cell contacts. Mol Biol Cell. 2009;20(7):1949-59.

24. Abufaraj M, Shariat SF, Haitel A, et al. Prognostic role of N-cadherin expression in patients with nonmuscle-invasive bladder cancer. Urol Oncol. 2017;35(5):264-71.

25. Nakajima S, Doi R, Toyoda E, et al. N-cadherin expression and epithelial-mesenchymal transition in pancreatic carcinoma. Clin Cancer Res. 2004;10(12 Pt 1):4125-33.

26. Kuphal S, Bosserhoff AK. Influence of the cytoplasmic domain of E-cadherin on endogenous Ncadherin expression in malignant melanoma. Oncogene. 2006;25(2):248-59.

27. Hazan RB, Phillips GR, Qiao RF, Norton L, Aaronson SA. Exogenous expression of N-cadherin in breast cancer cells induces cell migration, invasion, and metastasis. J Cell Biol. 2000;148(4):779-90.

28. Gerhardt H, Betsholtz C. Endothelial-pericyte interactions in angiogenesis. Cell Tissue Res. 2003;314(1):15-23.

29. Mariotti A, Perotti A, Sessa C, Rüegg C. N-cadherin as a therapeutic target in cancer. Expert Opin Investig Drugs. 2007;16(4):451-65.

30. Satelli A, Li S. Vimentin in cancer and its potential as a molecular target for cancer therapy. Cell Mol Life Sci. 2011;68(18):3033-46.

31. Dissanayake SK, Wade M, Johnson CE, et al. The Wnt5A/protein kinase C pathway mediates motility in melanoma cells via the inhibition of metastasis suppressors and initiation of an epithelial to mesenchymal transition. J Biol Chem. 2007;282(23):17259-71.

32. Cano A, Pérez-Moreno MA, Rodrigo I, et al. The transcription factor snail controls epithelialmesenchymal transitions by repressing E-cadherin expression. Nat Cell Biol. 2000;2(2):76-83.

\section{Figures}


A

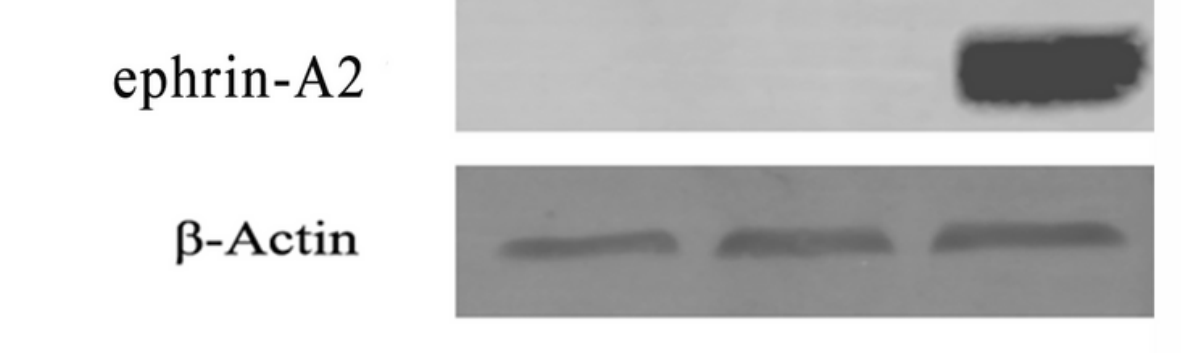

B

$\mathrm{C}$

ephrin-A2

GAPDH
$\mathrm{PC}-3 \quad \mathrm{PC}-3 / \mathrm{NC} \quad \mathrm{PC}-3 / \mathrm{A} 2$

\section{DU145}

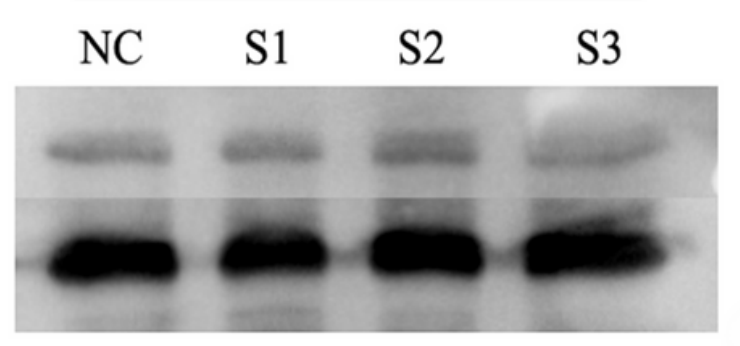

LNCaP

$\begin{array}{llll}\mathrm{NC} & \mathrm{S} 1 & \mathrm{~S} 2 & \mathrm{~S} 3\end{array}$
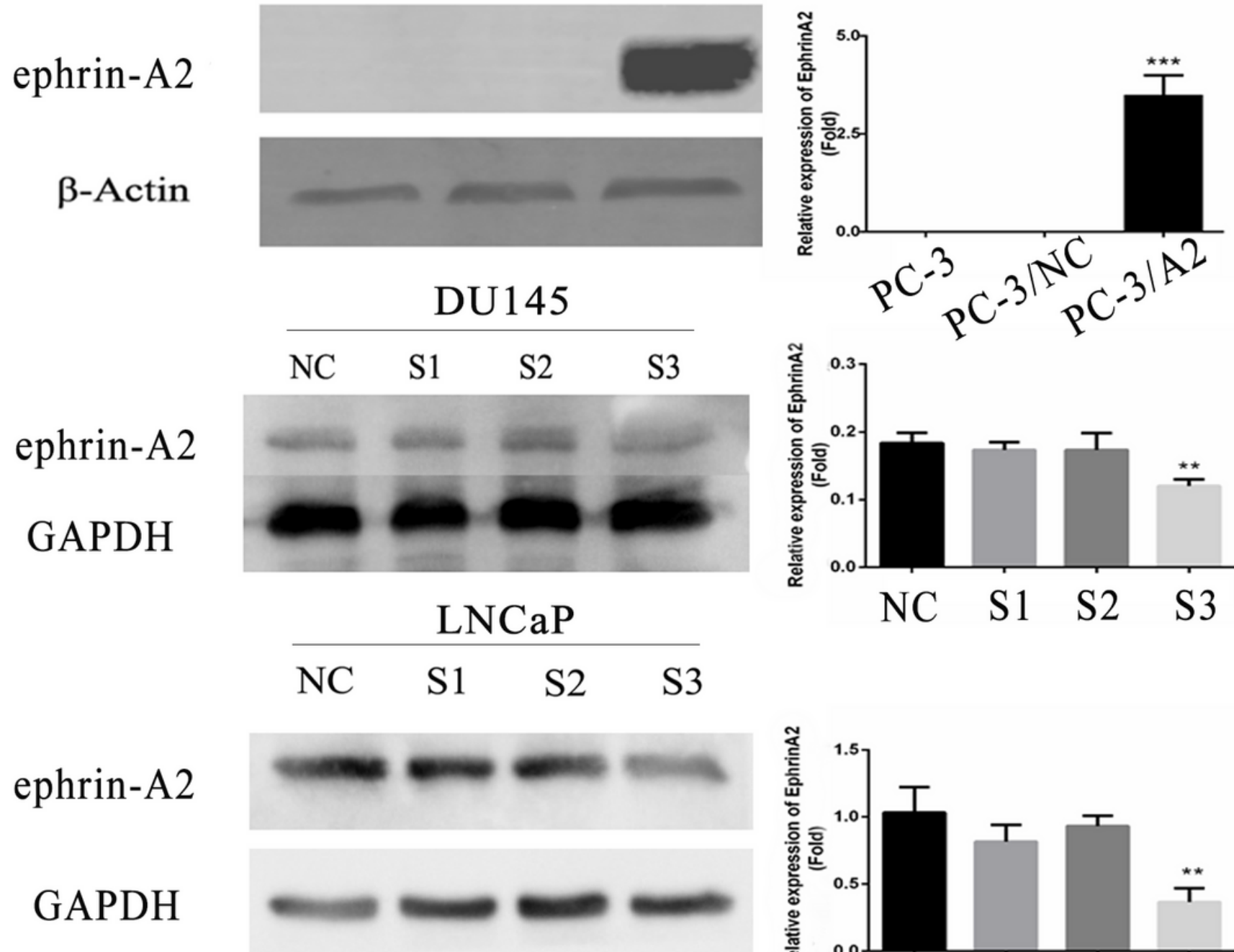

\section{Figure 1}

Ephrin-A2 was assessed in established stable cell lines through Western blotting analysis: A. Ephrin-A2 level was detected in PC-3 cell lines (Left panel). The relative expression of ephrin-A2 was normalized to that in PC-3 cells (Right panel). B. Ephrin-A2 level was detected in DU145 cell lines (Left panel). Ephrin-A2 relative level expression was normalized to that in DU145/NC cells (Right panel) C. Ephrin-A2 level was detected in LNCaP cell lines (Left panel). Ephrin-A2 relative level expression was normalized to that in LNCaP/NC cells (Right panel). ${ }^{*}<0.05$ compared with the control group. 
A
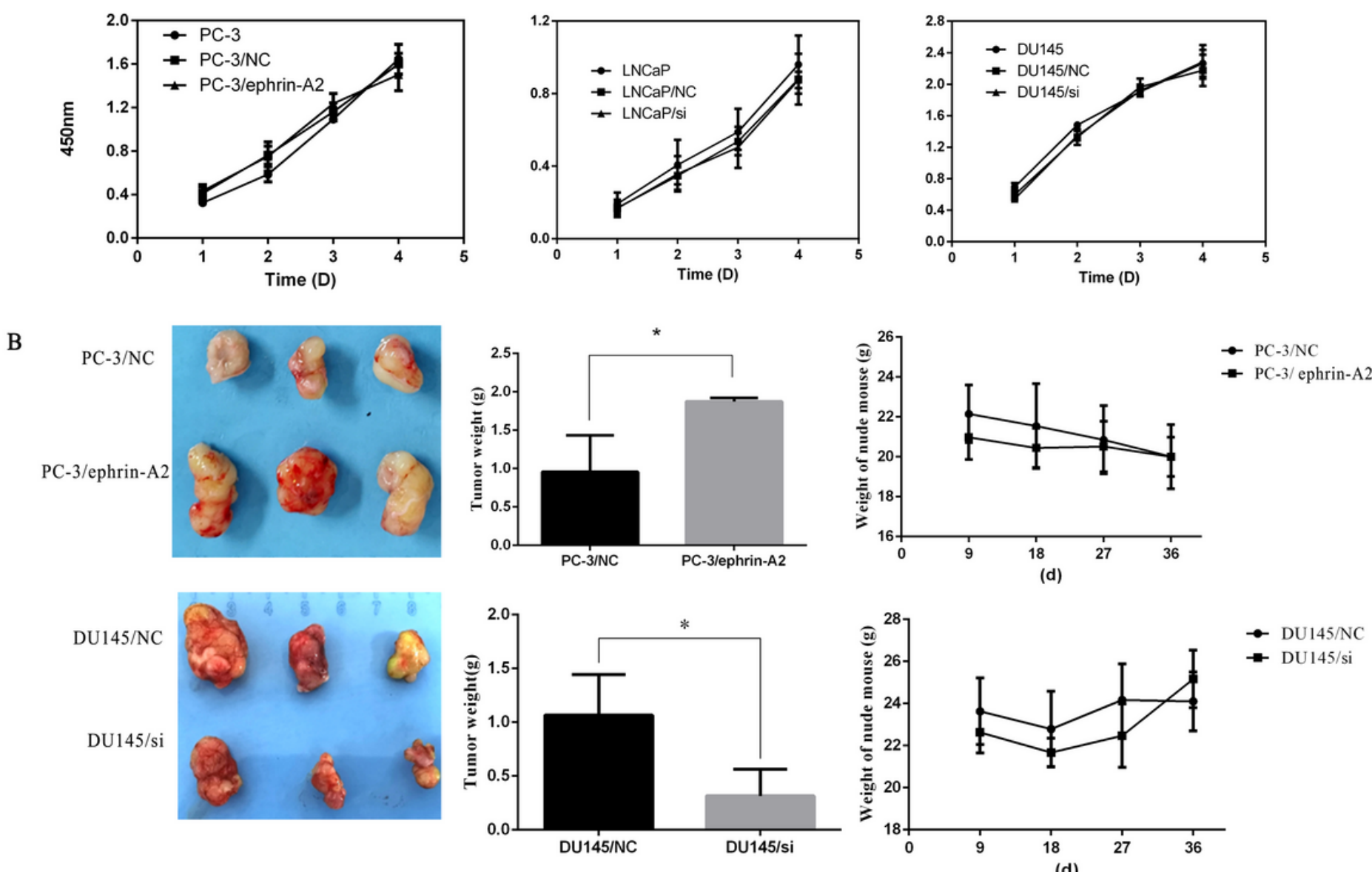

\section{Figure 2}

Ephrin-A2 contributed to tumor growth in stable cell lines in vivo: A. Ephrin-A2 showed no difference in cell proliferation according to the CCK-8 assay in vitro. B. Ephrin-A2 contributed to tumor growth in vivo. Tumor harvested from overexpressing ephrin-A2 PC-3 groups and ephrin-A2 knock-down DU145 group. 


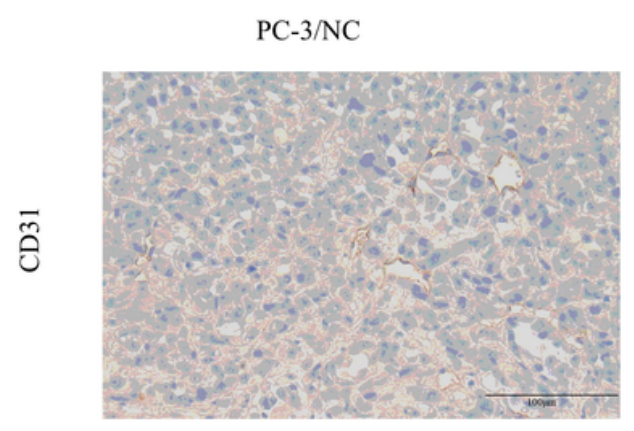

DU145/NC

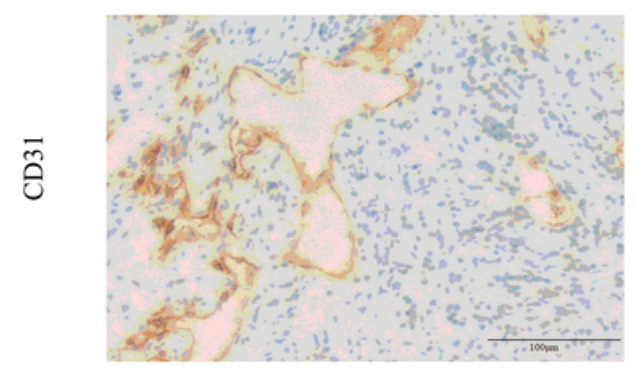

PC-3/ephrin-A2

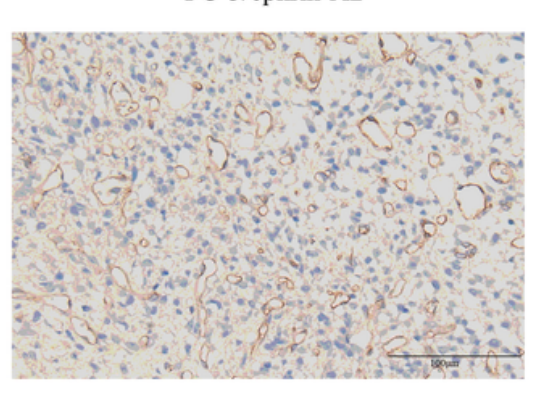

DU145/si

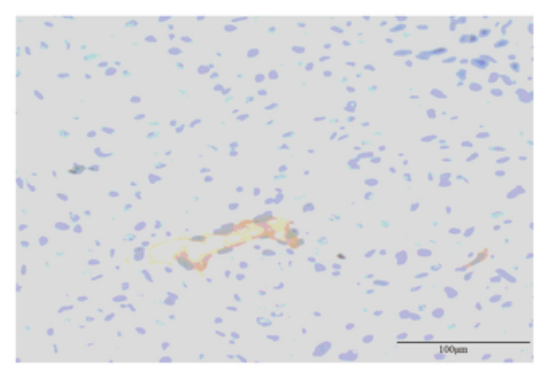

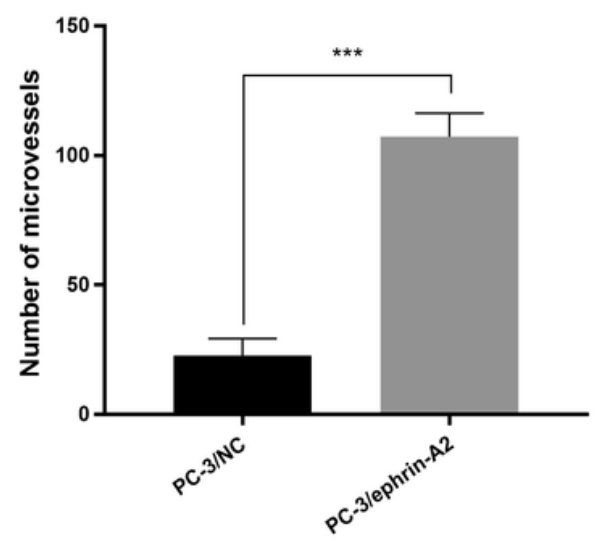

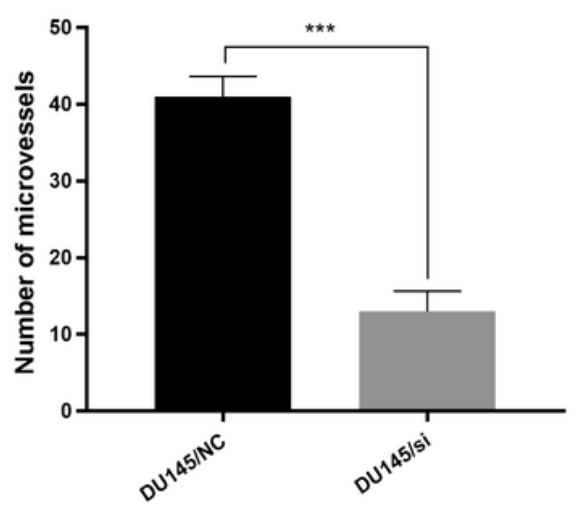

\section{Figure 3}

Ephrin-A2 promotes tumor angiogenesis. CD31 was evaluated in the xenograft models through immunohistochemical staining. Tissues harvested from overexpressing ephrin-A2 PC-3 groups and ephrin-A2 knock-down DU145 group. Scale bar, $100 \mu \mathrm{m}$. 
A

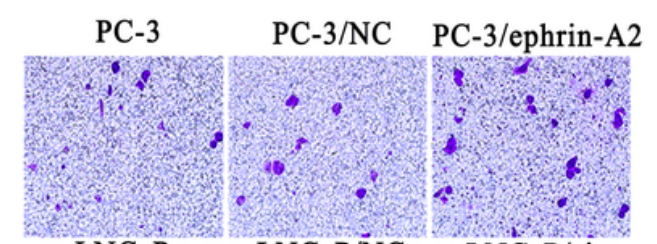

$\mathrm{LNCaP} \quad \mathrm{LNCaP} / \mathrm{NC} \quad \mathrm{LNCaP} / \mathrm{si}$

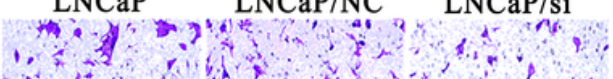

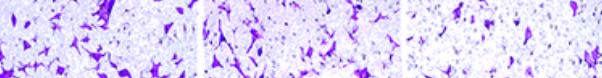

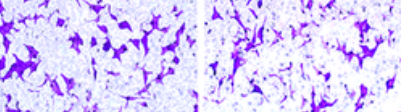

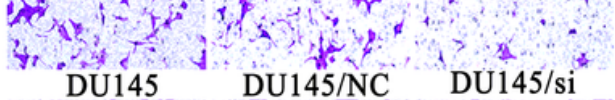

T. Pross DU145/NC DU145/si

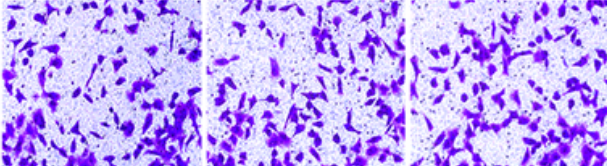

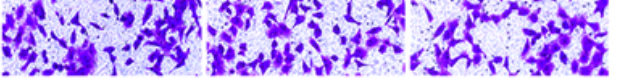

$\mathrm{C}$
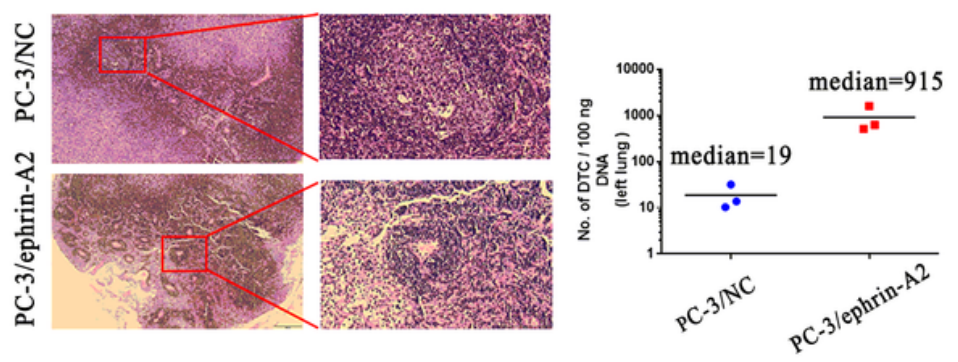

D
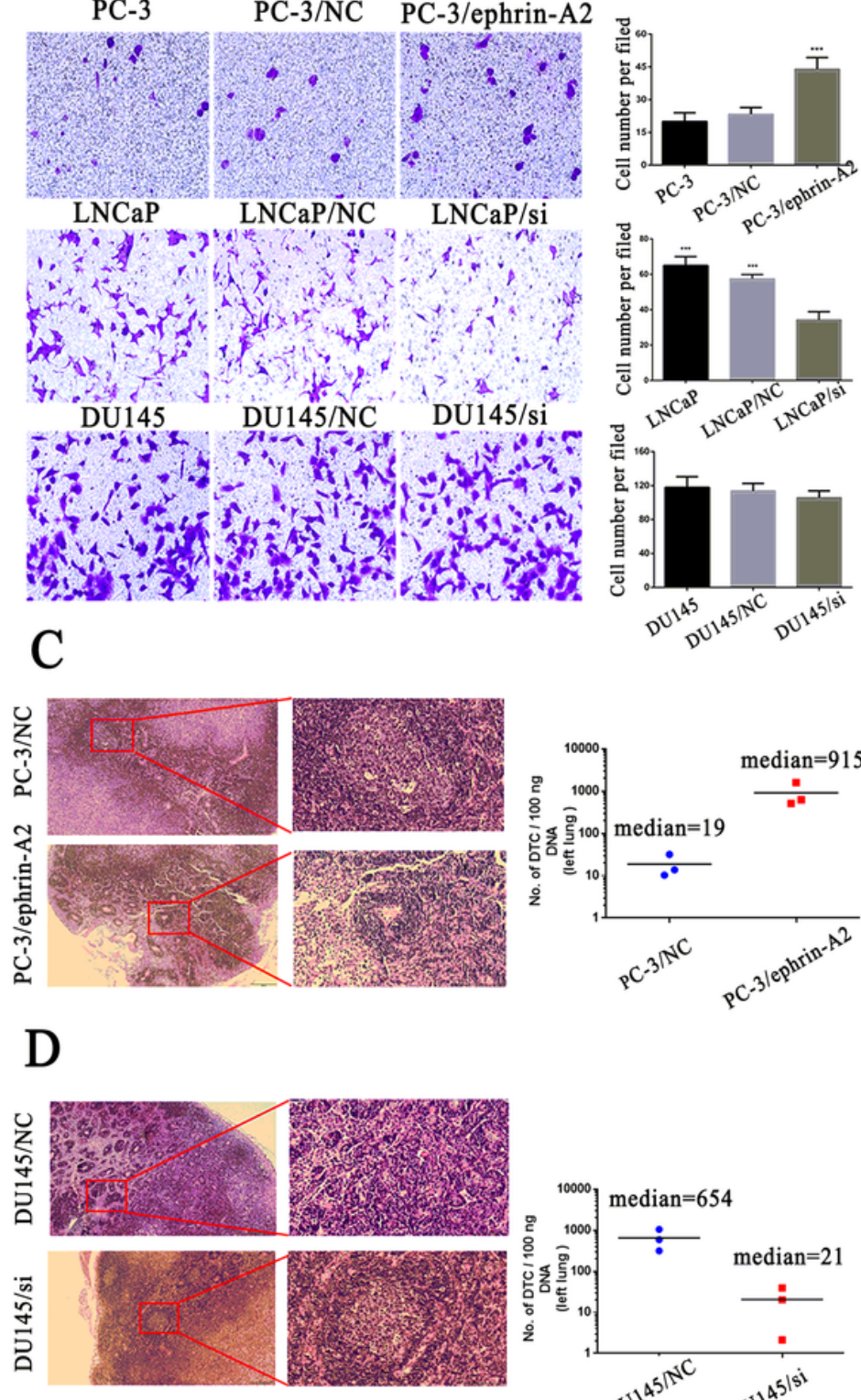

B
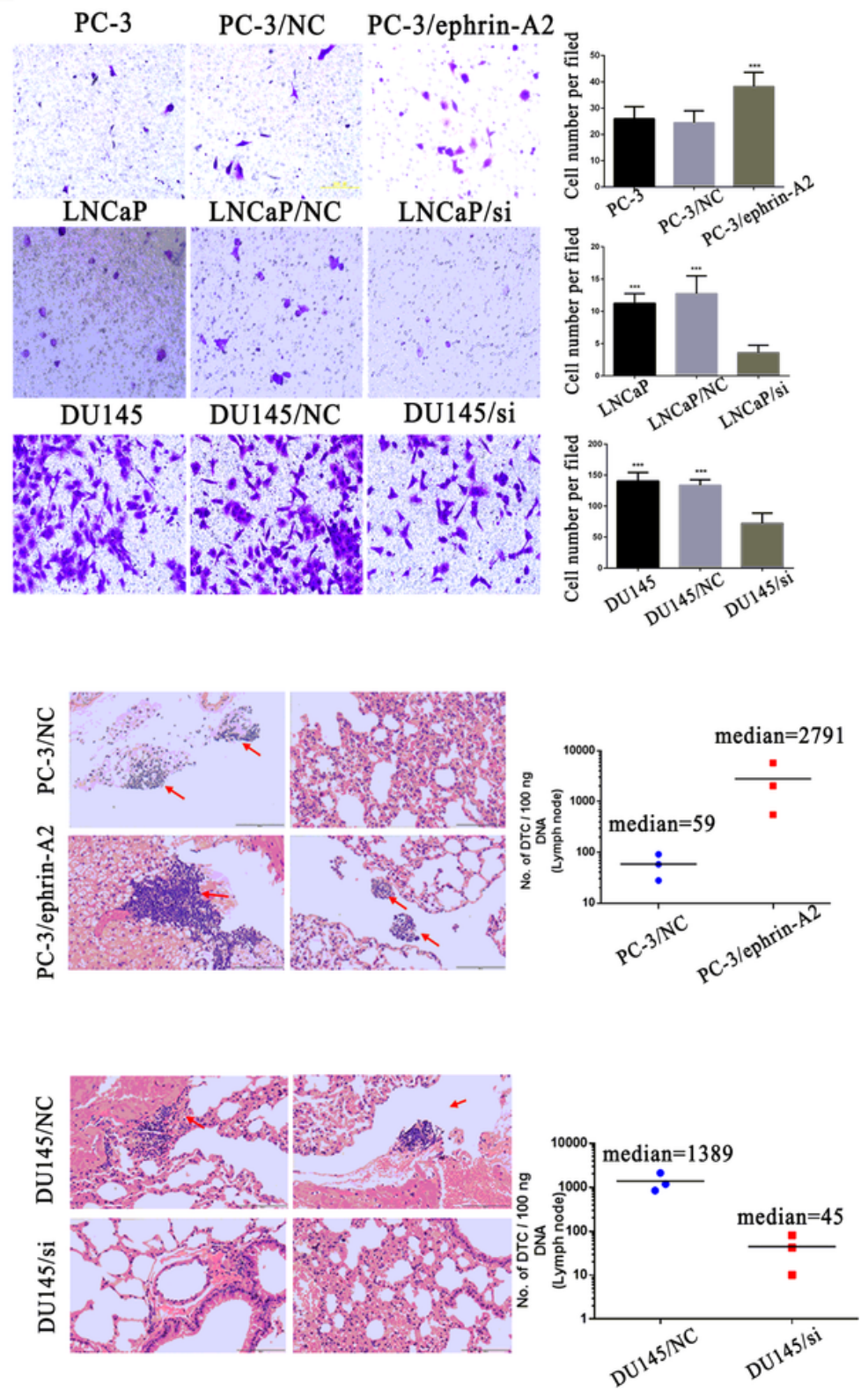

\section{Figure 4}

Ephrin-A2 led to tumor metastatic. A. The effect of ephrin-A2 on migration in three PCa cell lines. EphrinA2 enhanced PC-3 and LNCaP cell migration, but no effect on DU145. B. The effect of ephrin-A2 on invasion in three PCa cell lines. Ephrin-A2 enhanced prostate cell invasion. C\&D. Ephrin-A2 promote metastasis in vivo in PC-3 and DU145 group. Tissues were harvested and the existence of metastatic tumor cells in local draining lymph nodes (Left panel) and lungs (Right panel) were assessed by qPCRbased detection of human Alu sequences and HE staining. Scale bar, $100 \mu \mathrm{m}$. Scale bar, $100 \mu \mathrm{m}$. 

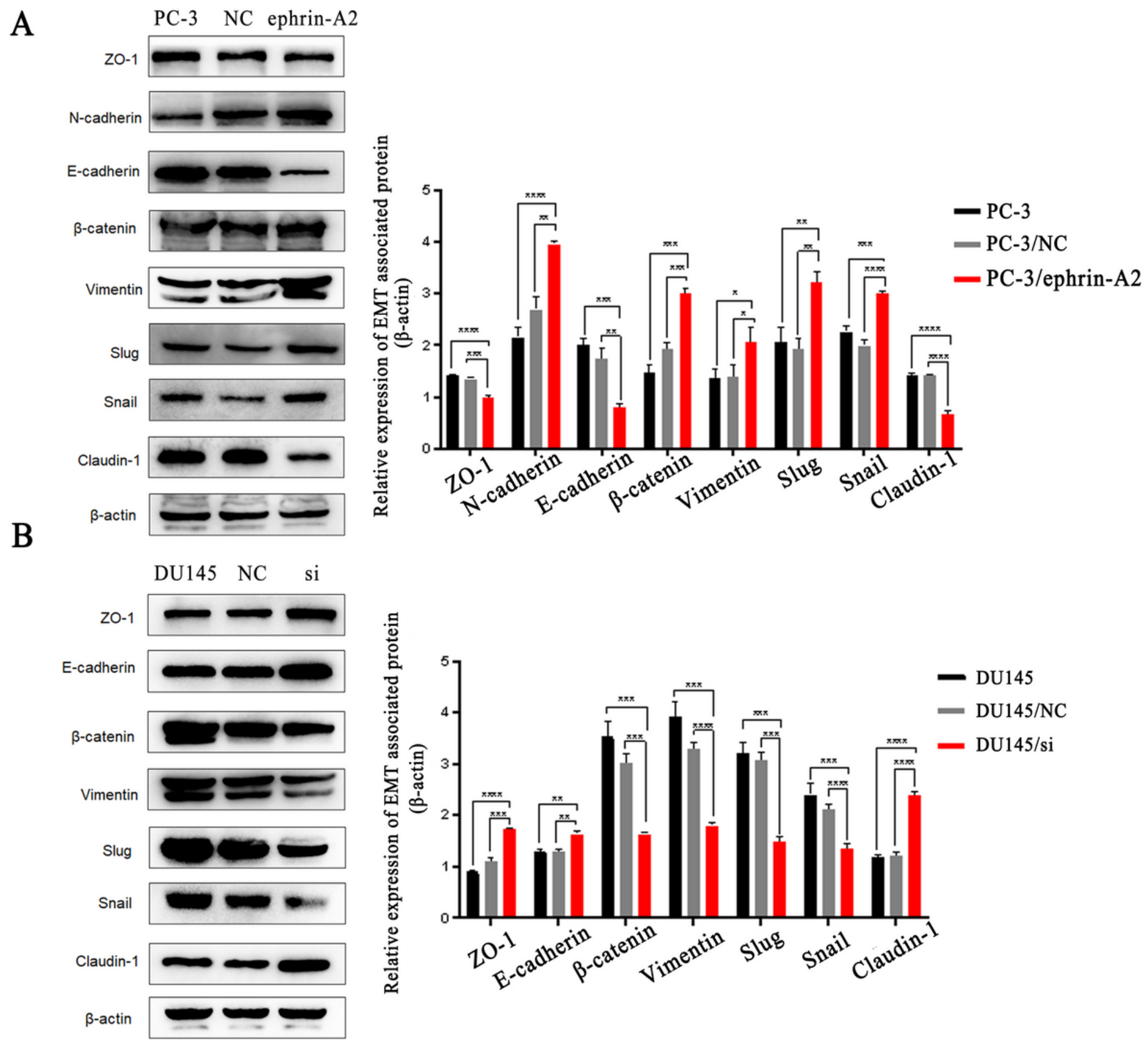

Figure 5

Ephrin-A2 altered the expression of EMT-related markers. A. Western blotting analysis of EMT-related markers protein expression in PC-3 cell lines (Left panel). The relative expression of ephrin-A2 was normalized to that in PC-3 cells (Right panel). B. Western blotting analysis of EMT-related markers protein expression in DU145 cell lines (Left panel). The relative expression of ephrin-A2 was normalized to that in DU145 cells (Right panel). 
A

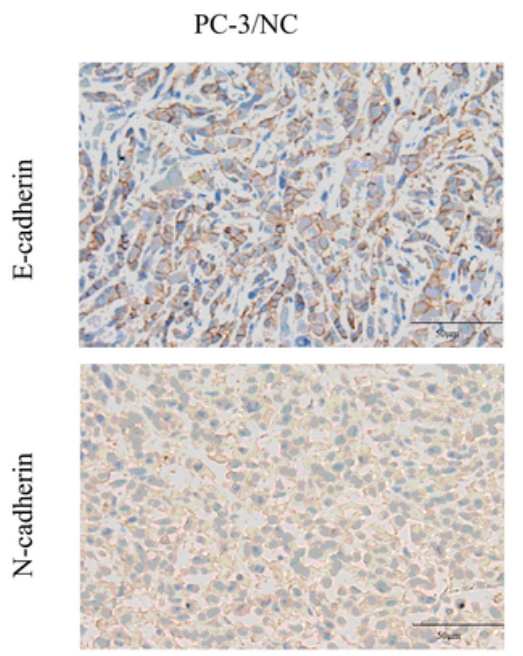

B

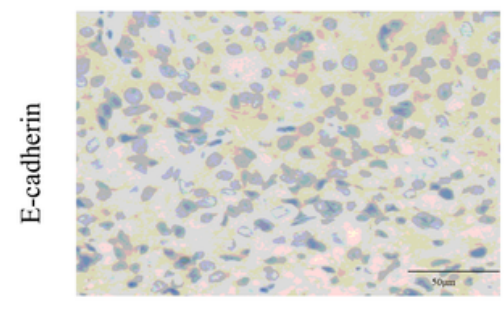

PC-3/ephrin-A2
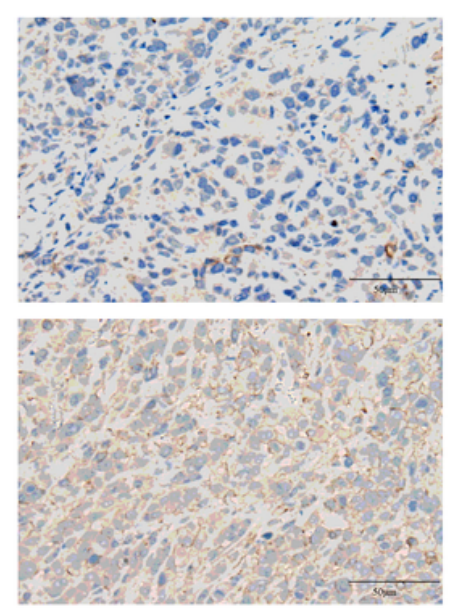

DU145/si

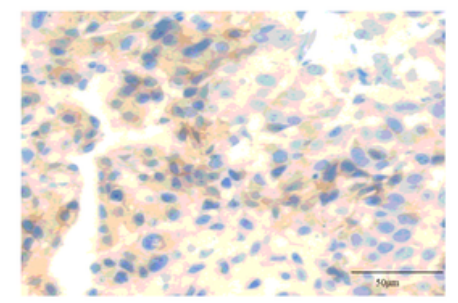

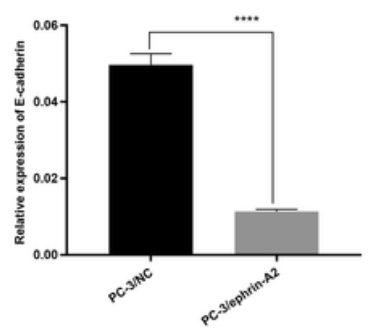
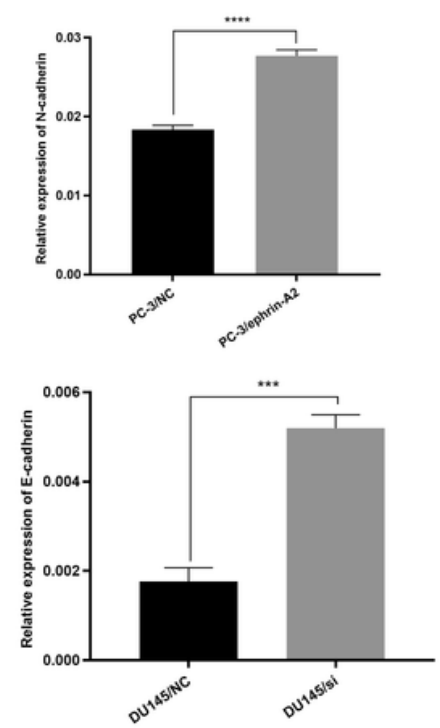

\section{Figure 6}

Ephrin-A2 affect the expression of E-cadherin and N-cadherin in xenograft models. A. E-cadherin and Ncadherin were analyzed in xenograft models by immunohistochemical staining. Tissues harvested from overexpressing ephrin-A2 PC-3 groups. Scale bar, $50 \mu \mathrm{m}$. B. E-cadherin were analyzed in xenograft models by immunohistochemical staining. Tissues harvested from knock down ephrin-A2 DU145 groups. Scale bar, $50 \mu \mathrm{m}$.

\section{Supplementary Files}

This is a list of supplementary files associated with this preprint. Click to download.

- SupportingDOC.docx

- SupportingDOC.docx

- SupportingFig.1.tif

- SupportingFig.1.tif

- SupportingFig.2.tif

- SupportingFig.2.tif

- SupportingFig.3.tif

- SupportingFig.3.tif 